\title{
SOME PROBLEMS OF INFERENCE IN TWO-DIMENSIONAL DISTRIBUTIONS
}

\author{
Dorothy Ann Anderson
}

This thesis is mainly concerned with problems of, and associated with, inference in some particular two-dimensional distributions. Making statistical inferences about parameters of a two-dimensional distribution is generally a very messy procedure. One tends to require simultaneous estimation of a number of parameters, and one needs to know the properties of these estimators. Often comparisons need to be made between sets of alternative estimators. For these comparisons such things as the bias, efficiency of estimation and ease of numerical calculation of the estimators are desired. In addition if the estimation equations are particularly messy the use of approximations may need to be considered. One often wants distributional and probabilistic properties of the twodimensional random vector to aid estimation of the parameters or obtain a better understanding of the two-dimensional distribution. Also the study of the estimation of the parameters of a two-dimensional random variable can often shed light on parametric estimation in an associated one- or multi-dimensional random variable, and vice versa.

Consequently although this thesis is mainly concerned with statistical inference in two-dimensional distributions that is not the only topic considered here. In Chapter 3 there is a discussion of infinite divisibility of bivariate gamma distributions; the motivation of which evolved from the work in Chapter 2 . Chapter 2 is concerned with estimation of the parameters of a particular class of bivariate distributions. One of

Received 27 July 1979. Thesis submitted to the Australian National University, June 1979. Degree approved December 1979. Supervisor: Professor P.A.P. Moran. 
the marginal distributions of this class is the exponential distribution, but the class does not include the gamma distribution. The work in Chapter 3 eliminates a possible line of inquiry as to how the analysis in Chapter 2 could be extended to the allied distribution with gamma marginals, and highlights some of the difficulties associated with this distribution. Also in Chapter 5 there is a discussion of the estimation of the noncentrality and scale parameters of a non-central chi distribution with known degrees of freedom. This evolved from the work in Chapter 4. The circular structural model is considered in Chapter 4. Although estimation in the general case is discussed there, due to its intractability more emphasis is placed on a special case. This involves a random variable which has a non-central chi distribution with unknown scale parameter and two degrees of freedom. Chapter 5 also explores the m-dimensional hyperspherical generalization of this circular model.

As indicated above, the circular structural model is examined in Chapter 4. One practical application of the circular structural model is to stone circle data and this is considered in Chapter 6. Some of the techniques actually used to estimate circular parameters are briefly mentioned. A circle fitting algorithm suggested for fitting stone circles is examined and compared with other estimation procedures.

When examining properties of estimators it is quite usual to be able to find only asymptotic properties, that is large sample properties. For example given certain regularity conditions a maximum likelihood estimator is asymptotically unbiased and, asymptotically, has a normal distribution with a specified variance. To find this variance we must be able to integrate the second derivative of the log-likelihood function. Techniques of numerical integration need to be employed if these integrals are not available from the literature. Such is the case in Chapters 2 and 4. Numerical integration of integrals also occurs in other contexts. For example in Chapter 3 numerical integration is used to describe the relationship between two variables.

Further, if other estimators of the parameters are available then by using the known efficiency properties of maximum likelihood estimators it may be possible to put a bound on the variance of the asymptotic distribution. This is explored in Chapter 4. 
However very often one might wish to make statistical inferences about parameter estimates that have been derived from a small sample. In a finite sample the distribution of the maximum likelihood estimator for example may not even have finite moments. Assuming the existence of moments we would like to know how large the sample size has to be before it can reasonably be assumed that the asymptotic properties of the estimator hold. A partial answer to this question can be obtained from simulation studies. In addition, for some of the estimators used no distributional properties can be derived, and only asymptotic moments can be found. We would like to know whether or not the distributions of any of these estimators can be assumed to be reasonably close to the normal distribution. Simulation studies can provide the answer to this question, as well as indicating whether or not any of the estimators have unexpected small sample properties. Some results of simulation studies carried out can be found in the second half of Chapter 6 .

Essentially only two different parametric estimation and inferential problems are considered in this thesis; a particular class of bivariate distributions, considered in Chapters 2 and 3 , and a circular structural model and allied models considered in Chapters 4,5 and 6. The problems associated with statistical inference are similar in both cases, and some of these problems have been described above. This thesis also illustrates the fact that a minor modification of a model can increase the mathematical complexity by an order of magnitude. This can turn a model from which a moderate amount of information can be extracted into one for which practically no information can be obtained. 